\title{
Présentation en avant-première d'une méthode sommaire d'estimation des crues sur de petits bassins de métropole: la méthode SØCØSE
}

\author{
Preview of the SOCOSE method for rough discharge \\ estimation in small French catchments
}

\author{
Guy Oberlin \\ Maitre de Recherches à l'ORSTOM \\ Chef de la Division Hydrologie - Hydraulique Fluviale \\ du CTGREF, Antony (France)
}

Dans le cadre des relations "pluies-débits" cette note présente un travail en voie d'achèvement et qui concerne une synthèse des crues effectuée sur les données des Services Régionaux d'Aménagement des Eaux (S.R.A.E.), données disponibles sur la banque qui existe depuis plusieurs années au Service de l'Hydraulique de l'Agriculture et pour laquelle le C.T.G.R.E.F. a mis à disposition les méthodes qu'il connaît et une grande partie de ses moyens pendant plusieurs années.

Cette synthèse présentera différents résultats et ceux exposés aujourd'hui concernent la question à l'ordre du jour de cette session. On pourrait les résumer sous la formule "modèle simplifié pluie-débit", non pas au sens de la transformation de chaque pluie en débit, mais au sens global de l'estimation des quantiles de débit qui intéresscnt lc projeteur, débit décennal par exemple, en fonction d'une connaissance globale des pluies.

En d'autres termes, il s'agit de l'essai de réglage d'une méthode objective d'estimation des crues, en principe décennales. En fait, on peut aller en-deça ou au-delà de cette fréquence : on n'est pas strictement tenu à la fréquence décennale.

\section{Spécificité de la méthode présentée}

En matière de crues et d'estimation de crues, il existe déjà quelques méthodes que nous connaissons et utilisons. Elles sont assez performantes au niveau de l'estimation, mais elles ont l'inconvénient de prendre en compte des paramètres relativement subjectifs, comme l'estimation d'une perméabilité de bassin.

Il nous est apparu que le créneau à remplir au niveau d'une méthode générale était plutôt le créneau objectif, puisque des méthodes en partie subjectives existaient.

D'autre part, au niveau du projet, de la pratique quotidienne et de l'hydrologie locale, l'expérience que nous pouvons avoir d'un secteur donné, et qui donc est quelque peu subjective, est déjà utilisée : c'est le fameux "pifomètre" de l'hydrologue. Là encore, la méthode proposée n'a pas la prétention de remplacer cette pratique et cette expérience qui restent valables et nécessaires.

La méthode en question a simplement pour objectif de proposer une solution en principe valable pour l'ensemble du territoire. C'est une première estimation d'un débit de crue à l'aide de paramètres qui ne prêtent pas à discussion, exception faite bien entendu des inévitables erreurs d'estimation.

\section{Bassins observés utilisés}

La figure 1 localise les bassins qui ont servi à cette synthèse.

On voit en première approximation que la répartition n'est pas trop mauvaise. En seconde approximation, on perçoit bien entendu des creux et des zones d'accumulation. Le critère n'a pas pu être un critère de couverture optimale du territoire car, en matière d'hydrologie, la qualité des données est essentielle. On a donc demandé à chaque S.R.A.E. de fournir, si possible, un nombre moyen (une dizaine ou une vingtaine) de bassins, mais nous n'avons retenu que ceux pour lesquels les données paraissaient de qualité suffisante, ainsi que ceux pour lesquels les durées d'échantillon étaient, sinon confortables, du moins supérieures à un seuil en-dessous duquel il n'était plus très sérieux de faire des statistiques (de l'ordre de 4 á 6 ans).

Il est utile de se rappeler ici qu'en matière de crues, l'information statistique est plus abondante qu'en matière de variables annuelles étant donné que les nombreuses crues d'une même saison d'hiver ont une certaine indépendance entre elles, ce qui permet, non pas de rallonger les durées, mais d'étoffer les échantillons statistiques. 


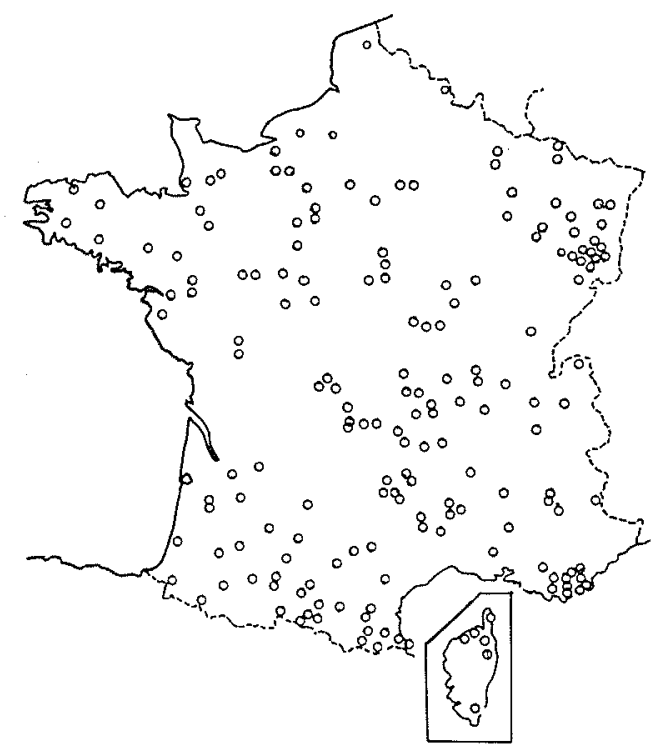

Figure 1 - Plan de situation des B.V. étudiés pour la méthode $S \emptyset \mathrm{C} \emptyset \mathrm{SE}$

\section{Choix des variables représentatives des crues}

Sur quelles données a-t-on travaillé ?

Outre que l'on cherchait à rendre cette méthode strictement objective, on s'est dit qu'il fallait essayer d'aller au-delà de l'estimation du débit de pointe et, si possible, aborder l'aspect "durée", ou plus exactement les questions pratiques posées au niveau "volume". Dans un premier temps, on a pensé étudier, d'une part, les débits et, d'autre part, le volume. Mais, dès qu'on regarde d'un peu plus près la question, on butte sur des difficultés parce que la variable "volume" est trop liée à la variable "débit".

On a donc très rapidement évolué et étudié comme seconde variable la variable "temps". En effet, les temps sont plus ou moins indépendants des débits et les deux notions sont suffisantes pour définir, sinon une forme d'hydrogramme de crue, du moins pour permettre une estimation du volume.

Après bien des réflexions, et après essais d'un certain nombre de variables déjà utilisées dans la littérature existante, on a choisi le temps pendant lequel la moitié du débit maximal était dépassé, $t_{s}$. C'est une variable qui a l'avantage de permettre d'estimer un hydrogramme triangulaire à partir des courbes habituelles (Fig. 2).

Que faire avec cette définition lorsqu'on se trouve devant des crues complexes? Le premier réflexe avait été de sommer les durées au débit maximal moitié. Puis, après quelques mois d'analyse, on est arrivé à des impasses et l'on est revenu, quand on nous présentait une crue complexe, à n'utiliser que la durée relative à la pointe principale en débit.

L'autre variable choisie a été le débit maximal $Q_{x}$, cela va de soi.

Pour le choix des crues, il y a eu des recherches de choix automatique sur les fichiers, mais il n'est pas très facile de régler un programme de choix automatique de crues qui a tendance à passer d'un extrême à l'autre : retenir presque toutes les crues ou alors oublier des

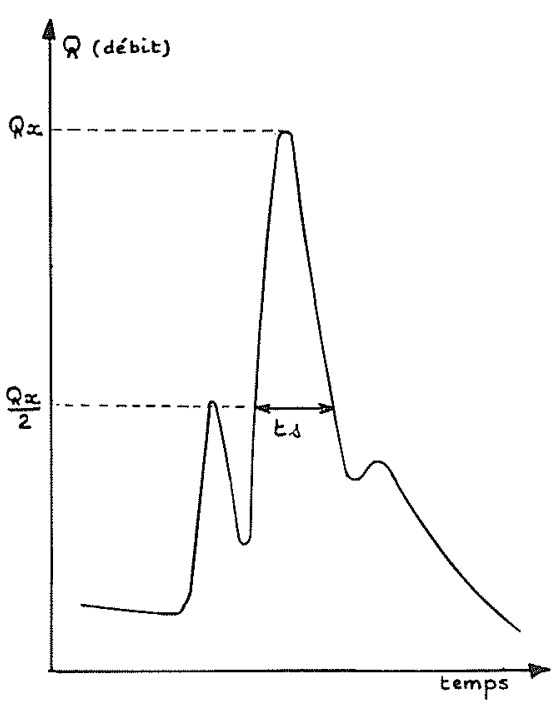

Figure 2 - Définition de la variable $t_{s}$.

crues intéressantes. Finalement le critère retenu a été : les 20 à 30 crues supérieures à un seuil en débit de pointe (aux lacunes près, bien entendu).

$\mathrm{La}$ relative indépendance entre les deux variables choisies, $t_{s}$ et $Q_{x}$, est illustrée sur la figure 3, qui représente les données d'un bassin. Il y a toutefois, dans ce cas, une légère décroissance du temps avec le débit. Ceci correspond à ce que nous connaissons tous, à savoir que les crues faibles sont souvent "molles" alors que les crues très importantes, dues à des phénomènes pluvieux exceptionnels, sont en moyenne un peu plus pointues que les autres. Avec le type de régression de la figure 3 , on prend le temps qui correspond au débit concerné : si l'objectif de l'étude est le débit décennal de pointe, on lui associe la durée $D$ correspondant à cette moyenne des temps liée aux débits.

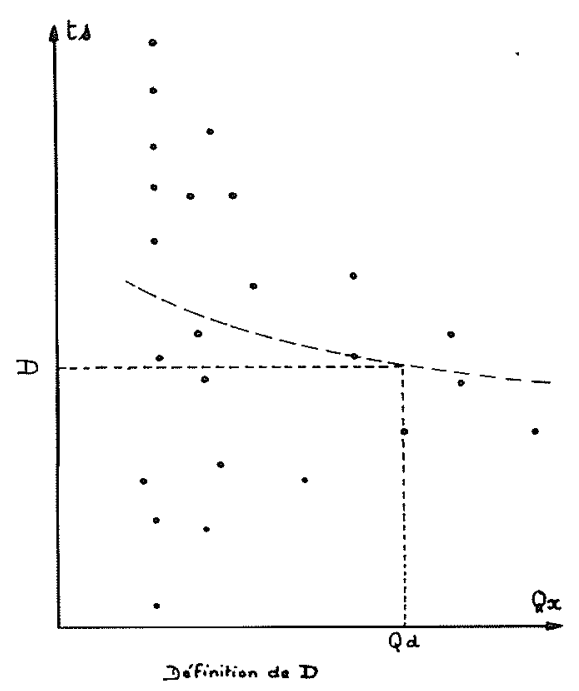

Figure 3 - Représentation des crues dans un plan. 


\section{Analyse et réglage du modèle}

La figure 4 présente l'organisation générale qui a été suivie pour le calage de la méthode $S \emptyset \mathrm{C} \emptyset \mathrm{SE}$, et la figure 5 quelques détails sur ce réglage.

Il est peut être utile de préciser ici d'où vient le nom de $\$ \emptyset C \emptyset S E$. C'est Robert Hlavek qui le premier l'a utilisé pour un modèle programmé au C.T.G.R.E.F. et qui s'inspirait de très près de la méthode dite du S.C.S. (Soil Conservation Service) du Département de l'Agriculture des Etats-Unis. C'était aussi un peu en hommage à $Y$. Cormary qui l'avait utilisé en Tunisie il y a de nombreuses années. La méthode présentée ici s'est beaucoup éloignée de ses origines mais le nom a été gardé.

Pour utiliser le modèle (Fig. 6), on dispose des données morphométriques et climatiques. Par des régres. sions multivariables celles-ci permettent d'estimer le paramètre temps $D$ et un paramètre de rétention $J$. De plus, les connaissances de la pluie locale permettent de définir une pluie de projet (paramètres $a$ et $b$ des formules de Montana).

De l'ensemble de ces résultats intermédiaires on déduit, grâce à l'utilisation de la fonction de ruissellement et de l'hydrogramme unitaire spécifique de la méthode $S \emptyset C \emptyset S E$, le débit décennal de pointe. En y associant le paramètre $D$ précédemment défini, on obient donc une estimation de l'hydrogramme.

Le réglage du modèle a consisté à inverser à peu de chose près les procédures précédentes. On est parti de données hydrologiques concernant les $n$ crues observées et choisies selon les critères cités précédemment. Une étude statistique a été effectuée sur ces $n$ couples $\left(Q_{x}, t_{s}\right)$. On en a déduit des valeurs "décennales" de $D$ et de $Q_{d}$. Un calcul à rebours du modèle a permis de définir le $J$. Ce $J$ et ce $D$ ont ensuite été expliqués par des régressions fonction de diverses variables du bassin. Celles-ci ont évidemment été choisies dans le lot des données climatiques et morphométriques citées précédemment.

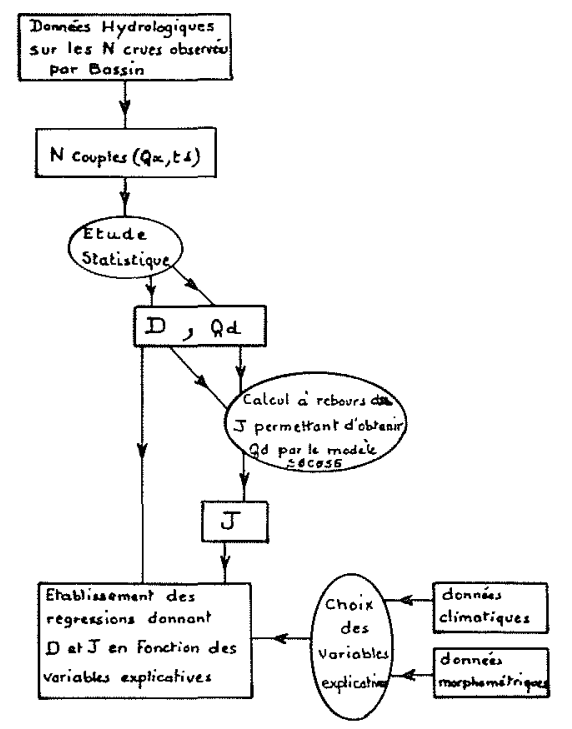

Figure 4 - Calage de la méthode SøCØSE. Organigramme d'ensemble.
(1) Sur $N$ annites dobstreration,

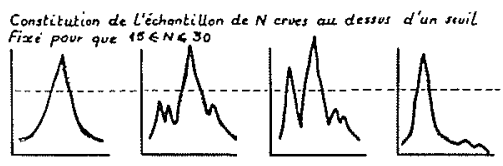

(2) Determination pour chaque crue du couph $(h x, t-1)$

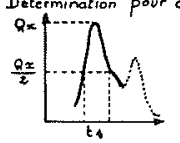

(3)

A juttement statifigue sur l'echantillon des $q_{z}$ et

determination du QD (en supposons lo prosecus paisonien)

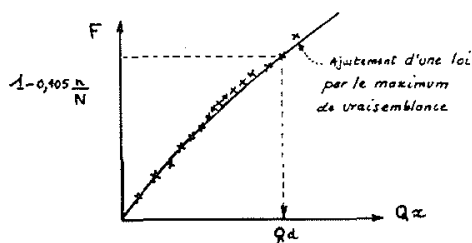

(4) Analyse dex graphigue des couptes (Q4, to) pour le calcut de $D$.

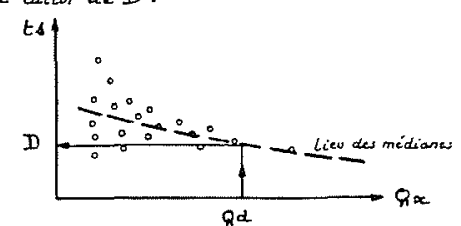

(5) Détermination itérative de $I$ por l'utilisation à rebours do madile socose

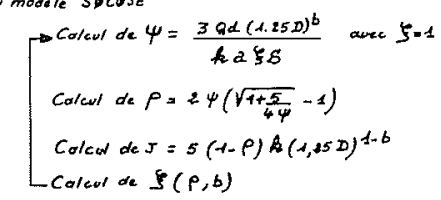

Arrä des calculs sidawx valeurs sactessives de

Fo diffeient de moins de 0.04 .

(6) Recherche des meilleures variables explicatives das grandaurs Der J.

Etablissement des regressions linegires comespondants

Figure 5 - Calage de la méthode $S \emptyset \mathrm{C} \emptyset \mathrm{SE}$. Description détaillée des opérations.

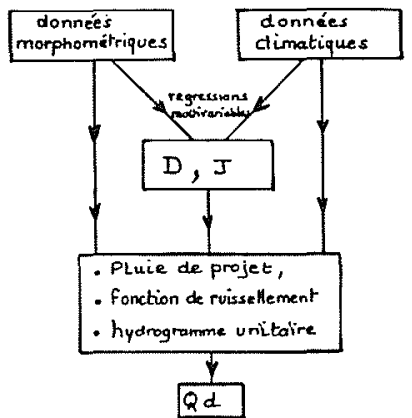

Figure 6 - Organigramme simplifié de la méthode S $\emptyset \mathrm{C} \emptyset \mathrm{SE}$.

La figure 5 détaille un peu les procédures, en particulier celles concernant l'inversion des modèles pour la détermination de $J$.

Il a été dit que $J$ est une espèce de rétention. C'est plutôt un indice de rétention et d'infiltration et, quoiqu'il s'exprime en $\mathrm{mm}$, il ne faut pas trop le comparer 
à d'éventuelles mesures qui pourraient être effectuées sur les bassins. Son ordre de grandeur est en particulier fonction de la forme de la fonction de rendement qui est spécifique du modèle $S \emptyset C \emptyset S E$.

Dans les variables explicatives, grande a été la surprise de devoir abandonner successivement la plupart des données morphométriques : malgré le désir de les voir figurer, il n'était pas possible de les rendre significatives.

\section{Analyse des résultats}

La figure 7 présente les deux formules de base donnant $D$ et $J$. A l'origine, nous souhaitions raisonner de manière aussi déterministe que possible. Ainsi, $D$ devait être initialement une somme de trois temps, chacun d'eux étant une fonction de ce qui dans un bassin est sensé déterminer les vitesses d'écoulement. Comme cela a été dit plus haut cet objectif n'a pu être atteint et pourtant les moyens dont nous disposions étaient considérables. En particulier, le Service de 1'Hydraulique de l'Agriculture avait mis à notre disposition l'ensemble du fichier morphométrique établi dans les bassins des S.R.A.E., notamment pour optimiser l'implantation du réseau des stations. Ce fichier morphométrique avait été élaboré dans des conditions relativement satisfaisantes de normalisation. Il est considérable puisqu'il atteint la cinquantaine de caractéristiques par bassin. Malgré cette information, on a dû abandonner, et la forme de la somme, et la plupart de ces caractéristiques. Comme on peut le constater figure 7 , ne figurent plus que la surface $\mathrm{du}$ bassin, la pluie journalière décennale locale, la pluie annuelle moyenne et la température annuelle moyenne ramenée au niveau de la mer. Nous étions loin des variables initialement prévues.

L'importance de l'échantillon de réglage ne permet pas de mettre en cause la significativité de ces relations. Il faut en conclure que la plupart des paramètres morphométriques qui ont fleuri ces dernières années dans la littérature hydrologique, ne sont visiblement pas adaptés à l'explication des débits, du moins en zone rurale.

Des problèmes analogues ont été rencontrés dans l'ex. plication de $J$. On constate que la formule reprend à peu de choses près les mêmes paramètres que ceux de $D$, à cette différence près que la températurc annuclle est remplacée par la longueur du thalweg. On constatera

$\ln (D)=-0.69+0.32 \ln (S)+2.2 \sqrt{\frac{P a}{P} \frac{1}{t a}}$
$J=260+21 \ln \left(\frac{S}{L}\right)-54 \sqrt{\frac{P a}{P}}$

avec:

$D$ en heures

$J$ en $\mathrm{mm}$

$S$ en $\mathrm{km}^{2}$ : Superficie du B.V.

$L$ en $\mathrm{km}$ : longueur du Thalweg le plus long

$P$ en $\mathrm{mm}$ : pluie journalière locale décennale

ta en ${ }^{\circ} \mathrm{C}$ : températion moyenne interannuelle réduite

au niveau de la mer

$P a$ en $\mathrm{mm}$ : pluie annuelle moyenne, en $\mathrm{mm}$

Figure 7 - Résultats des régressions statistiques. (Résultats provisoires)

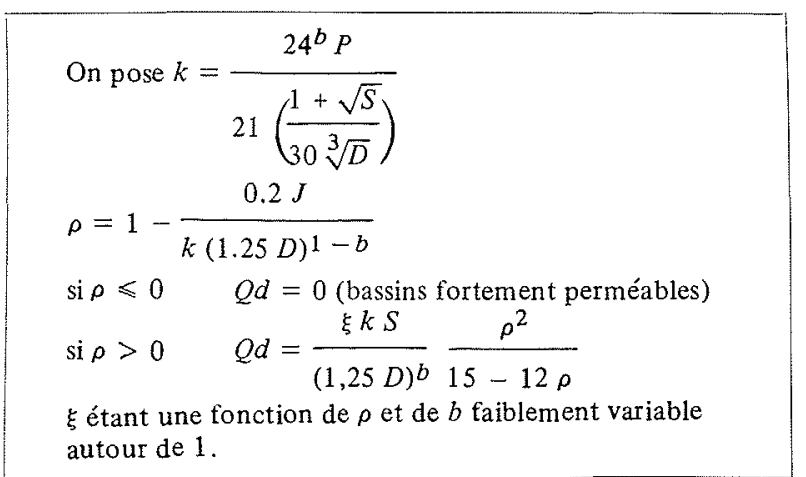

Figure 8 - Résultat brut de la méthode $S \emptyset \mathrm{C} \emptyset \mathrm{SE}$.

(Résultats provisoires).

que cette longueur du thalweg n'intervenait pas dans la variable temps. Ceci est a priori étonnant ; mais il est impossible dans un réglage de modèle de forcer la significativité ou la non-significativité d'une variable, quels que soient les raisonnements physiques que l'on puisse tenir à ce sujet.

La figure 8 complète les formules précédentes en donnant le détail du calcul des deux variables intermédiaires $k$ et $\rho$ et l'estimation de $Q_{d}$ en conséquence. Le lecteur reconnaitra dans le $k$ l'intervention des lois de Montana et du phénomène de l'abattement, et dans $\rho$ l'intervention de la fonction de rendement spécifique du S.C.S. et qui est un peu différente des formules habituelles de Horton ou issues de celle de Philips.

La complexité relative de ces formules, l'existence des variables intermédiaires $k$ et $\rho$ et celle du coefficient de correction $\xi$, ont pour origine le seul souci de rendre la méthode plus opérationnelle, c'est-à-dire d'éviter à l'utilisateur de faire autre chose que des calculs simples et des lectures d'abaques. $\mathrm{Ne}$ pas exprimer la procédure pratique sous cette forme, aurait exigé de la part de l'utilisateur de réaliser, pour chaque estimation, les divers calculs d'intégration que nécessite cette méthode du type hydrogramme unitaire (Fig. 9).

\section{Quelques détails sur les fonctions de rendement et de transfert}

L'allure de la fonction de rendement est représentée sur la figure 10: sa formulation s'éloigne de celle de Horton mais la figure parle d'elle-même et montre une relation de type asymptotique qui est tout à fait habituelle pour ce genre de modèle.

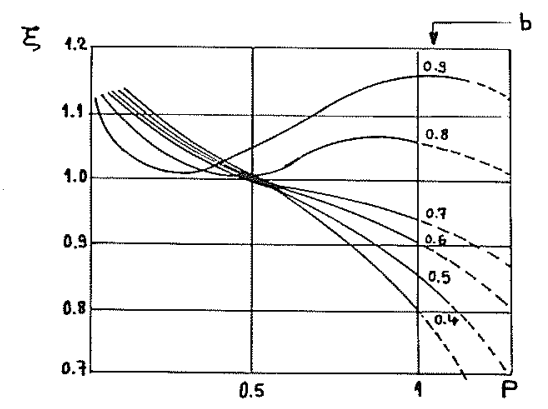

Figure 9 - Graphique de $\zeta$. 


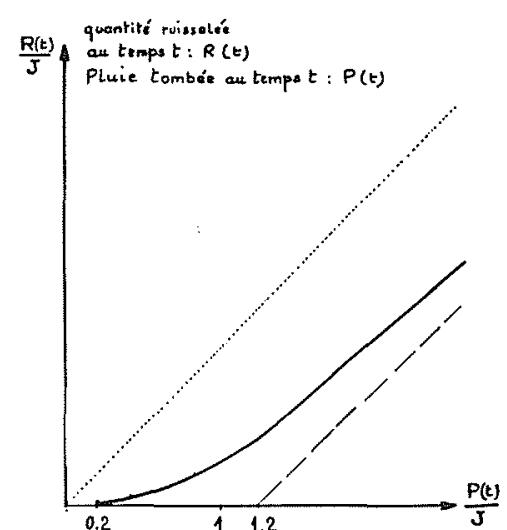

Figure 10 - Fonction de rendement.

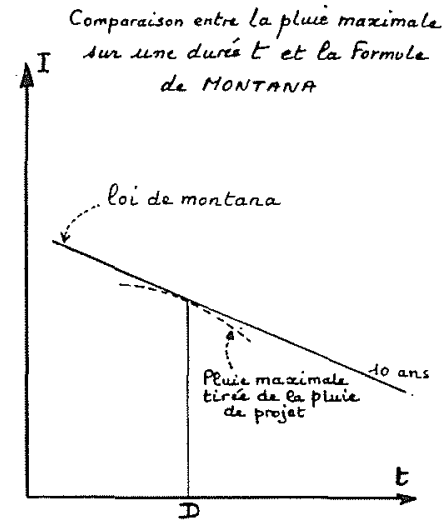

Figure 11 - Hyétogramme de projet.

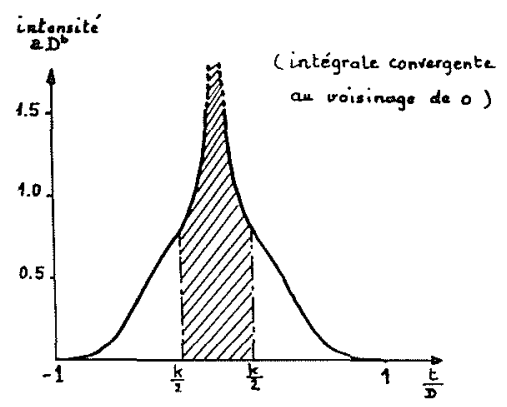

Fig. 12. - Hyétogramme de la pluie de projet de la méthode S $\varnothing \mathrm{C} \emptyset \mathrm{SE}$. (La superficie hachurée est notée $P(k, D)$ ).

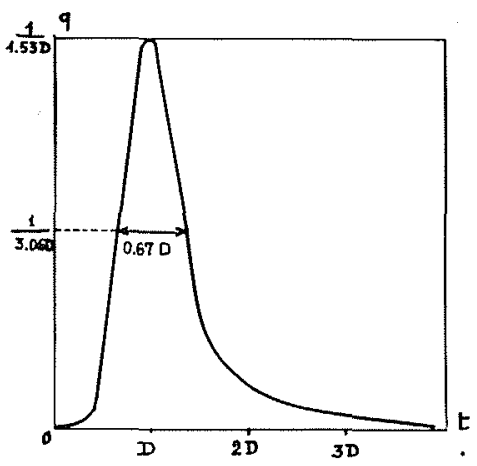

Fig. 13 - Hydrogramme unitaire de la méthode $S \varnothing \mathrm{C} \emptyset \mathrm{SE}$.

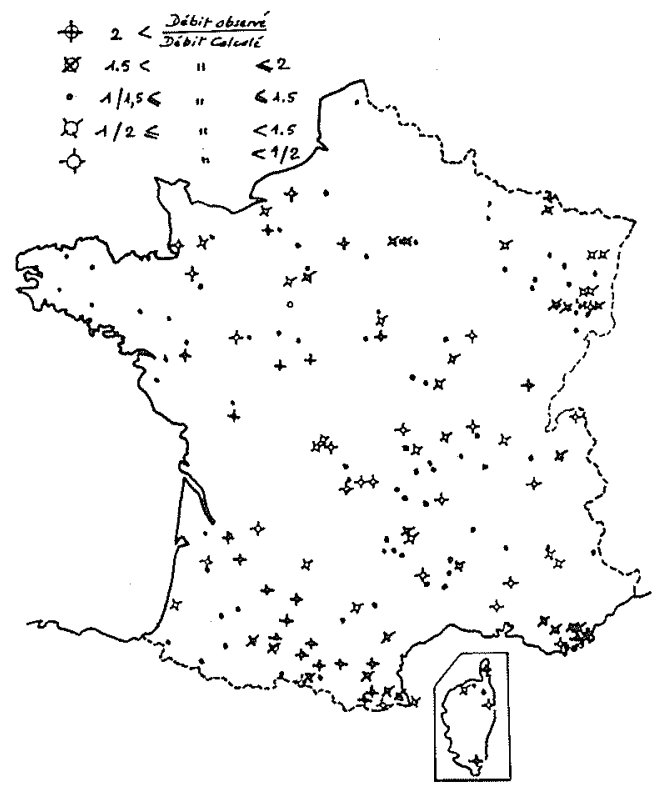

Fig. 14 - Débit maximal de crue. Comparaison entre les valeurs observées et les valeurs calculées pour les Bassins versants étudiés.

La figure 11 présente la façon dont varient les intensités des hyètogrammes de projet par rapport aux lois de Montana. Pour une fréquence principale (ici décennale), on constate que les fréquences des durées supérieures et inférieures sont légèrement moins rares et ne s'éloignent que progressivement de la décennale.

La forme du hyétogramme (Fig. 12) est originale mais a été choisie aussi pour des raisons pratiques de calcul. Quant à l'hydrogramme unitaire (Fig. 13) il ne diffère d'un triangle que pour les raisons citées ci-dessus et son allure est classique.

\section{Contrôle des résultats}

La figure 14 illustre les écarts constatés entre les valeurs observées et les valeurs estimées par la méthode S $\varnothing \mathrm{C} \emptyset \mathrm{SE}$. Il s'agit de la variable $Q_{d}$, débit maximal de crue, de fréquence décennale. On constatera que les écarts sont moyens pour une bonne moitié des bassins, mais assez importants pour une part non négligeable de l'échantillon. De plus, certaines zones ne sont pas exemptes d'une erreur quasi systématique, comme par exemple le bassin de la Garonne.

Il faut rappeler à cette occasion que cette méthode est strictement objective. Ceci signifie qu'aucun des paramètres n'a pu être "réglé" de façon à améliorer l'adéquation du modèle. En ce sens, il ne faut pas juger ce modèle avec les mêmes critères que ceux qui concernent des modèles plus subjectifs.

Il résulte de ceci une bonne homogénéité dans les estimations et un ordre de grandeur de l'erreur connu et identique pour tout le monde. Qu'elle soit relativement forte n'étonnera aucun hydrologue : cette méthode ne change rien à ce que nous savons depuis longtemps, c'està-dire qu'en hydrologie les observations sont irremplaçables et donnent très rapidement, sous réserve qu'elles 
soient de qualité convenable, des résultats bien meilleurs que les méthodes d'estimation, aussi sophistiquées soient elles.

\section{Conclusion}

La synthèse nationale des crues des petits bassins dont il a été question ici verra ses résultats publiés d'ici quelques mois et le modèle $S \emptyset \mathrm{C} \emptyset \mathrm{SE}$ décrit dans cette note représentera un des fascicules de cette synthèse. Un autre fascicule concernera une méthode plus géographique, qui donnera des résultats à caractère régional et dont la précision sera approximativement la même que celle de $S \emptyset C \emptyset S E$. Un autre fascicule détaillera les relations pluie-débit et les caractéristiques de crue, lesquelles devraient permettre un affinement local dans un certain nombre de cas où l'information pluie est bonne.

\section{Discussion}

M. DESBORDES. - Avez-vous effectué des comparaisons de vos résultats avec ceux obtenus en 1968 par SOGREAH à la suite d'une étude semblable, sans doute de moindre ampleur, et realisée pour le compte du Service des Autoroutes?

M. OBERLIN. - On n'a pas eu le temps de faire ce travail systématiquement. On a fait quelques tests çà et là. Peut-être que M. MAILHOL a quelques résultats sous la main puisqu'il fait un contrôle sur quelques bassins de longue durée ; mais je ne sais pas si les abaques SOGREAH ont été utilisés dans ces tests.

Ce que je peux dire, c'est qu'étant donné que nous avions à notre disposition une masse d'informations relativement importante, on a en principe des débits qui sont plutôt plus faibles. A la faveur des progrès en matière d'hydrologie de petits bassins, on arrive à baisser un peu les coefficients de sécurité et en moyenne à rabaisser les débits de crue estimés.

Sur quelques bassins où l'on faisait une erreur d'interprétation et où l'on n'avait pas perçu qu'il y avait là des pluies exceptionnelles ou un écoulement un peu plus fort que d'habitude, les nouvelles estimations sont en hausse mais. en général, les valeurs récentes sont plutôt inférieures.

Notre vœu aurait été d'aller au-delà des abaques SOGREAH et, non seulement de supprimer le paramètre subjectif, mais encore, in troduire les paramètres du réseau hydrographique pour que cette méthode puisse servir quand il $y$ a une influence humaine sur le réseau. Malheureusement nous n'y sommes pas arrivés bien qu'on se soit battu pour cela.

Alors, provisoirement, et malgré notre ambition du début, on ne peut pas utiliser cette méthode pour différencier, par exemple, deux bassins sur lesquels le réseau de drainage se développe. Il y a le thalweg principal, mais il n'y a pas la densité de drainage, il n'y a pas les "constantes" d'Horton, etc. Mais peut-être tout cela n'a pas vraiment d'influence sur les crues?

\section{THIRRIOT. - Deux questions :}

1) Quel est l'objectif spécifique de la fonction de ruissellement ?

2) Quelle est la méthodologie de construction des formules empiriques?

M. OBERLIN. - L'hydrogramme unitaire, ce n'est qu'une méthode de transfert

La partie compliquée de la méthode, c'est le passage de la pluie tombée à la pluie nette excédentaire, efficace au sens des hydrologues de surface. C'est le réglage de cette fonction qui a attiré notre attention.

Nous sommes partis de la fonction du S.C.S. et c'est parce que ce règlage s'est révélé délicat que nous n'avons pas cherché une autre fonction de rendement plus proche des équations d'infiltration, alors qu'initialement nous avions l'idée de prendre au moins Horton, sinon des équations tirées de Philips.

Ensuite, la transformation par un hydrogramme unitaire ne pose pas de problème et c'est pour des raisons purement diplomatiques que nous sommes passés du triangle à l'hydrogramme "naturel' que je vous ai montré.

En ce qui concerne votre seconde question, je suis embarrassé pour vous répondre, car le travail a été fait essentiellement par Claude MICHEL, qui n'a pas pu venir aujourd'hui.

Par scrupule j'ai dit que la durée aurait dû être la somme d'un certain nombre de termes qui, de façon déterministe, peuvent expliquer la durée, l'épaisseur d'une crue ou la durée du temps de réponse. Ce sont la Iongueur du réseau, les pentes dans la partie qui n'est pas encore réseau... les couvertures végétales (parce qu'elles in terviennent au niveau de la rugosité).

Mais il a été impossible de régler cela. même en puisant d'une façon réfléchie dans le lot des 50 paramètres disponibles. De fil en aiguille, on en est venu à prendre des paramètres de plus en plus éloignés de ceux initialement choisis pour en arriver à des choses comme la pluie annuelle!

D'autre part, on est passé en logarithme parce qu'on n'arrivait pas à trouver d'autres anamorphoses qui rendent les distributions marginales vaguement normales. Dans une version datant de 2 ou 3 mois, on avait une formule qui était aussi bonne que celle-là. qui n'était pas en logarithmes, mais les nuages de régression étaient tellement mauvais qu'on nous a suppliés de sortir des régressions ayant unc allurc plus normale. C'est ce qui nous a conduits in extremis et à contre-cœur à passer à une formule de logarithme.

M. THIRRIOT. - Et la coexistence des racines et des logarithmes?

M. OBERLIN. Quand les fonctions réagissent moins vite que la fonction linéaire, on essaie d'être cohérent. On prend des puissances inférieures à 1 ou des logarithmes. On a mis $D$ en logarithme pour gagner un peu d'explication de variance tout à la fin du règlage.

A partir du moment où l'on fait des calculs qui donnent à la méthode des allures de "recettes de cuisine" au niveau de l'utilisation, c'est une faute très mineure que de terminer le réglage par une introduction d'hétérogénéité.

M. GUILLOT. - Vous êtes partis d'un échantillon de 180 bassins.

M. OBERLIN. - M. SCHERER pourrait dire : $190 \ldots$

M. GUILLOT. - . . et vous avez dit que vous aviez fait des régressions. On a vu une formule de régression qui donnait le débit décennal. 
M. OBERLIN. - L'analyse de ces échantillons a été faite en en tirant une collection de débits maximaux et une collection de durées. Ce sont les épaisseurs des hydrogrammes à $Q_{x} / 2$. De l'analyse des $Q_{x}$ on a tiré une estimation de la décennale, avec tout l'intervalle de confiance qu'il faut lui donner, compte tenu de la faiblesse des échantillons. On a fait la même chose sur $Q_{d}$. plus un petit controle de l'indépendance.

M. GUILLOT. - Pour chacun des bassins vous connaissez le débit décennal ?

M. OBERLIN. - Oui, nous avons un $Q_{d}$ et un $D_{d}$.

$M$. GUILLOT. - La formule de régression donne $Q_{d}$

M. OBERLIN. - Le $Q_{d}$ est issu d'une méthode qui est à peu de chose près la méthode statistique classique. La régression concernait d'abord $D$ puis le paramètre $J$ d" "infiltration" obtenu par inversion de la méthode connaissant $D$ et $Q_{d}$.

Il n'y a de véritable régression isolée que pour $D$. Pour le reste il y a un réglage, mais vous ne pouvez pas le ramener à un règlage par multivariables.

M. GUILLOT. - Est-ce que vous pourriez donner les corrélations partielles de chacun des termes? Quels sont les termes les plus significatifs?

Il y avait la surface du bassin versant qui intervenait; ensuite il y avait un indice composite et tout cela en racine carrée. . . Ce deuxième terme en particulier, est-ce que ce n'est pas par gentillesse que vous l'avez conservé ?

M. OBERLIN. - Dans la méthode "hydrogramme unitaire", vous n'êtes pas libre d'isoler les variables. Quand vous discrétisez la transformation. compte tenu de la fonction de rendement (avec un numérateur au carré et un dénominateur linéaire) des complications sont déjà introduites. Au niveau du règlage du modèle, nous avions donc déjà des rapports de variables ou des produits.

Ensuite, quand une variable se révélait insuffisamment significative pour la mettre isolément avec son coefficient de régression, il nous est arrivé de la mettre de côté puis de la réintroduire dans une expression pour qu'elle joue quand même, selon son influence positive ou négative sur les débits. C'est ainsi que se sont introduites des variables composites.

Pour les coefficients de régression partielle, il faudra examiner les résultats détaillés qui seront publiés dans les fascicules cités. La regression totale entre $Q_{d}$ observé et $Q_{d}$ estimé donne un coefficient de corrélation de 0.83 .

M. SCHERER. - Je voudrais apporter un élément de réponse au sujet de la formule qui suscite des question de la part de M. GUILLOT.

Il s'agit de l'estimation de la durée de crue correspondant à un débit égal à la moitié du débit maximal.

Les paramètres qu'a livré l'analyse statistique sont : la surface, $\sqrt{P_{a} / P}$ et la température réduite au niveau de la mer. Ces deux dernières variables ont été retenues à la suite d'une analyse statistique et non a priori.

A la question de savoir pourquoi ces deux variables sont liées à la durée de la crue, on peut apporter la réponse suivante : le rapport "pluie annuelle/pluie journalière décennale" a une signification climatique. Il prend des valeurs voisines de 20 en Bretagne, de 15 à 20 dans le Bassin Parisien, de 4 ou 5 dans les Cévennes et de 6 ou 7 en Provence. Il a une structure semblable à celle de l'indice d'agressivité climatique de Fournier (précipitation mensuelle maximale au carré rapportée à la pluie an nuelle).

Il peut s'interpréter comme un indice permettant une différenciation climatique entre des régions "tempérées océaniques" et des régions "méditerranéennes".

Il n'y a pas le même type de pluies dans ces deux types de régions et cela influe sur la durée de la crue. C'est sans doute là l'explication de la présence des variables climatiques dans la regression.

M. GASOIOSKI. - Dans ce cas, est-ce qu'il ne vaudrait pas mieux régionaliser cette formule ?

M. OBERLIN. - Effectivement ce travail a été fait à l'échelle nationale parce qu'il fallait bien le commencer ; mais je crois que plusieurs Services Régionaux d'Aménagement des Eaux (c'est en tout cas ce que souhaite le Service hydrologique de l'Agriculture) ont maintenant pour objectif de poursuivre le travail quand ils auront les moyens de réaliser cette synthèse, en y ajoutant peut-être alors d'autres bassins... Entre-temps, on aura heureusement gagné quelques années.

Pour compléter la réponse de M. SCHERER, il faut dire que le $D$ qu'on a ici, n'a presque plus rien à voir avec les temps de concentration déterminés par les formules de Passini et autres. Ils sont beaucoup plus grands. Nous avons dû réviser notre idée de temps caractéristique de bassin : les quelques heures qu'on trouvait sur les petits bassins n'ont que peu de signification pratique. Les crues d'été aussi bien que d'hiver, en ce qui concerne les petits bassins, même pour les plus pointues d'entre elles, ont des durées plus longues que ce que donnaient les formules type Turazza. Cela explique que les paramètres climatiques finissent par entrer dans quelque chose qui n'est plus une durée de transfert hydraulique, mais qui intègre toutes sortes de choses.

Ceci dit, il a fallu que M. SCHERER insiste beaucoup pour que nous introduisions ces données. On ne le voulait pas. On a dû finalement changer d'avis pour être raisonnable vis-à-vis des coefficients de corrélation.

M. GUILLOT. - J'ai en tête un article américain paru il y a plusieurs années où un responsable important du Service hydrométrique américain U.S.G.S. avait fait une analyse statistique des crues sur un important échantillon de bassins. Son propos était de savoir si la connaissance de l'information pluviométrique avait un intérêt quelconque pour estimer les débits extrêmes. Sa conclusion était totalement négative.

L'écueil dans ce genre d'approche est qu'on mélange trop de facteurs.

M. OBERLIN. - Notre vœu est que cette méthode remplace des méthodes qui étaient moins bien réglées et surtout moins objectives en matière de première estimation des crues. Il est bien évident qu'à la limite on souhaiterait qu'elle ne serve que comme terme de comparaison.

Il faudrait que sur un projet donné on fasse l'estimation avec cette méthode. Puis qu'on fasse cette estimation et une étude hydrologique sur une station hydrologique proche. Cette méthode ne servirait alors en quelque sorte que de "règle de 3" pour transférer le résultat de la station sur le lieu du projet.

Nous ne souhaitons pas qu'elle soit utilisée isolément, sauf bien entendu dans les cas où l'on ne peut pas faire autrement, ou dans les cas ou économiquement une étude n'est pas justifiée.

M. GHIO. - Je ne suis pas tout à fait d'accord avec M. OBERLIN sur le problème de temps de concentration. J'avais appliqué les formules italiennes et cela m'avait donné des résultats différents de ceux qu'on observait sur de petits bassins. Ce n'est pas un critère absolu, mais cela ne me paraissait pas parfait.

M. OBERLIN. - M. GHIO a acquis une bonne expérience locale de ce problème et a appliqué une méthode sommaire en réglant des coefficients. Mais quelqu"un qui "débarque" dans la région Centre et qui chercherait à appliquer la méthode Turazza ne fera que des bêtises. Au contraire, avec cette méthode, quelqu'un qui "débarque" est assuré de ne pas totalement dérailler. 